\title{
Loop-induced photon spectral lines from neutralino annihilation in the NMSSM
}

\author{
G. Chalons ${ }^{a}$ and A. Semenov ${ }^{b, c}$ \\ ${ }^{a}$ Institut für Theoretische Teilchenphysik, \\ Karlsruhe Institute of Technology, Universität Karlsruhe, \\ Engesserstraße 7, 76128 Karlsruhe, Germany \\ ${ }^{b}$ Laboratory of Particle Physics, Joint Institute for Nuclear Research, \\ 141980 Dubna, Moscow Region, Russian Federation \\ ${ }^{c} L A P T H$, Université de Savoie, CNRS, \\ BP 110, F-74941 Annecy-le-Vieux Cedex, France \\ E-mail: guillaume.chalons@particle.uni-karlsruhe.de, \\ semenov@lapp.in2p3.fr
}

ABSTRACT: We have computed the loop-induced processes of neutralino annihilation into two photons and, for the first time, into a photon and a $Z^{0}$ boson in the framework of the NMSSM. The photons produced from these radiative modes are monochromatic and possess a clear "smoking gun" experimental signature. This numerical analysis has been done with the help of the SloopS code, initially developed for automatic one-loop calculation in the MSSM. We have computed the rates for different benchmark points coming from SUGRA and GMSB soft SUSY breaking scenarios and compared them with the MSSM. We comment on how this signal can be enhanced, with respect to the MSSM, especially in the low mass region of the neutralino. We also discuss the possibility of this observable to constrain the NMSSM parameter space, taking into account the latest limits from the FERMI collaboration on these two modes.

KEYWORDS: Supersymmetry Phenomenology

ARXIV EPRINT: 1110.2064 


\section{Contents}

1 Overview of the NMSSM 3

2 Set-up of the automatic calculation $\quad 5$

3 Additional NMSSM contributions $\quad 6$

4 Benchmark points and comparison with the MSSM $\quad 7$

4.1 SUGRA 8

$\begin{array}{llr}4.2 \text { GMSB } & 10\end{array}$

5 Spectral lines from light neutralino annihilation $\quad \mathbf{1 2}$

$\begin{array}{lll}5.1 & \text { Light singlet pseudoscalar Higgs resonance } & 12\end{array}$

5.2 Gamma-ray lines as a further constraint on the NMSSM parameter space. 15

A NMSSM BRST transformations for the scalar fields

Introduction. The existence of cold dark matter (CDM) is supported by many geometrical and dynamical observations coming from cosmology and astrophysics, but its detection needs to be confirmed. Its properties, such as mass, spin, and interactions are still to be determined. A long term experimental effort has been devoted to its detection, from direct methods, where a dark matter particle is expected to impinge on a nuclei in a target material, or more generally from the products of its self-annihilation in outer space (indirect detection).

Recently the CDMS [1], CoGeNT [2, 3] experiments and very recently CRESST [4] have pointed out results whose explanation could be interpreted as hints in favour of the existence of low mass dark matter particles, although the recent result of the XENON100 disfavors this possibility [5] and the CDMS-II results seems to be in contention with CoGeNT [1]. The interesting piece of information is that these results could be reconciled with the long standing claims of the DAMA/LIBRA experiment that dark matter was detected through its annual modulation [6]. However these potential signals of dark matter are still an open debate and no firm claim of dark matter signal can be established, since there is no general agreement between the different experiments. If a dark matter hypothesis is to be put forward to interpret these signals, the studies points to a low mass WIMP. Thus some attempts were made to account for them, for example with neutralinos lighter than, say, $15 \mathrm{GeV}$. In recent publications the authors of [7-12] considered and investigated the occurrence of light neutralino within the Minimal Supersymmetric Standard Model (MSSM) and Next-to-Minimal Supersymmetric Standard Model (NMSSM) while respecting several constraints. It was shown that an MSSM explanation for the signal is seriously challenged by collider searches and measurements in the flavour sector $[7,8,13,14]$. 
The Next-to-Minimal Supersymmetric Standard Model is a well-motivated extension of the MSSM constructed by adding a singlet superfield $\hat{S}$ in the Higgs sector. This has been advocated to provide an elegant solution to the so-called $\mu$ problem of the MSSM but it also renders the Higgs "little fine tuning problem" less severe [15]. Moreover it leads to a richer spectrum than the MSSM: two additional Higgs bosons (one CP-even and one CP-odd neutral Higgs) remain after ElectroWeak Symmetry Breaking (EWSB) and the neutralino sector is enlarged by a fifth neutralino. This last possibility has important consequences concerning dark matter studies, that can differ significantly from the MSSM, especially when the lightest neutralino composition is dominated by the singlet component, the singlino. Moreover the occurrence of very-light singlet pseudoscalar in the Higgs spectrum and at the same time evading the LEP bounds is still possible thanks to reduced couplings to standard model particles. These facts open new possibilities in relic density calculation as well as direct detection prospects as opposed to the MSSM. In particular the thermally produced neutralinos can satisfy the measured CDM present relic abundance while being very light. This implies that the pair annihilation rate of thermal relics roughly scales as $1 / m_{\chi}{ }^{2}$. All these observations make a light supersymmetric candidate for dark matter more plausible in the NMSSM than the MSSM. It is then appealing to investigate the self annihilation of dark matter in our galaxy since one expects light neutralinos to give significantly enhanced rates for the indirect detection signals compared to the standard case.

Numerous studies have been devoted to relic density and direct detection phenomenology in the NMSSM, few work has been dedicated to indirect detection of dark matter (annihilation of a pair of dark matter particle) through the "direct" annihilation into primary monochromatic photons [16]. These primaries photons have the advantage of being less affected by astrophysical uncertainties over other kind of messengers. Moreover their spectrum would reveal a sharp peak at an energy $E_{\gamma} \simeq M_{\chi}$ corresponding to the mass of the dark matter particle for the $\gamma \gamma$ final state and $E_{\gamma} \simeq M_{\chi}\left(1-M_{Z}^{2} / 4 M_{\chi}^{2}\right)$ for $\gamma Z^{0}$, since in the galactic halo $v / c \simeq 10^{-3}$. Provided one has a good enough detector energy resolution and sensitivity, the flux from these primaries photons will be clearly distinctive from the astrophysical background or diffuse emission. However modeling the dark matter halo is still needed since the number density of dark matter particles enters the calculation. The FERMI collaboration did not report any line observation and has released upper limits on the direct annihilation $\langle\sigma v\rangle_{\gamma \gamma}$ and $\langle\sigma v\rangle_{\gamma Z}$ [17] instead. The recently installed AMS [18-20] detector on the International Space Station may also shed light on these channels.

The complete computation of the loop-induced annihilation into photons has already been performed in [21-25] for the MSSM. In [16] the one-loop amplitudes for NMSSM neutralino pair annihilation into two photons and two gluons have been given (adapted to the NMSSM case from the formulas given in [21, 22]) and prospects for the indirect detection of the monochromatic gamma-ray line were also investigated. In light of the latest limits of the FERMI collaboration on monochromatic gamma ray signal and the recent activity in the dark matter community, we propose to revisit the two gammas mode and provide for the first time results on the $\gamma Z^{0}$ one. We investigated to which extent the NMSSM rates could differ from the MSSM and for the numerical study we focused on 
mSUGRA and GMSB benchmarks points given in [26, 27]. This is a case study if one wants to discriminate the NMSSM from the MSSM with dark matter related observables, which basically means to which amount the neutralino sector is sensitive to the extended Higgs sector. We further explored in which case, specific to the NMSSM, the spectral line can be enhanced and if such mechanisms can be constrained by astrophysical measurements. Constraining these mechanisms is very interesting since it also impacts the relic density calculation and direct detection predictions.

These rates were computed with the help of the SloopS program, an automatic code for one-loop calculation in the context of the SM and the MSSM [28, 29]. This code has already been used for accurate relic density predictions at next-to-leading order in the MSSM [30$32]$ and also to the numerical computation of the indirect channels $\tilde{\chi}_{1}^{0} \tilde{\chi}_{1}^{0} \rightarrow \gamma \gamma, \gamma Z^{0}$ and two gluons mode [25]. Therefore this work is also a good exercise to test the implementation of the NMSSM in SloopS. On the technical level, as the dark matter particles are moving at relatively small velocity, the Gram determinant, which is a key ingredient for calculating the loop integrals, vanishes and this results in numerical instabilities. This has been handled with a procedure called segmentation [25]. We will come back to the numerical and technical details of the implementation later. The outline of the paper is as follows, in section one we quickly review the NMSSM model and its parameter space, in section two we describe the SloopS code and the implementation of the NMSSM. A particular attention will be paid to the implementation of non-linear gauge-fixing in the NMSSM. The third section will be devoted to scrutinise the additional contributions brought when going from the MSSM to the NMSSM. In the fourth section we will give the rate for the $\gamma \gamma$ and $\gamma Z^{0}$ channels for some SUGRA and GMSB scenarios and compare it to an equivalent MSSM spectrum. In the following section we will discuss on some possible ways of increasing the signal, specific to the NMSSM, and discuss the relevance of using the sharp gamma lines as a constraining observable, taking into account the latest published limits. Finally we will draw our conclusions.

\section{Overview of the NMSSM}

In the NMSSM the Higgs term of the superpotential involving the two Higgs doublet is modified and a singlet term is added, ${ }^{1}[15]$

$$
W_{N M S S M}=W_{M S S M}^{\mu=0}+\lambda \hat{S} \hat{H}_{u} \hat{H}_{d}+\frac{\kappa}{3} \hat{S}^{3}
$$

The MSSM $\mu$ bilinear term is now absent from the superpotential and has been replaced by the trilinear coupling of the singlet with the two Higgs doublets. The VEV of the singlet generates an effective $\mu$ parameter with respect to the MSSM, which is then naturally of order the EW scale [15],

$$
\mu_{\mathrm{eff}}=\lambda s
$$

\footnotetext{
${ }^{1}$ We stick to the " $\mathbb{Z}_{3}$-invariant NMSSM", where any dimensionful parameters in the superpotential are forbidden.
} 
where $s=\langle\hat{S}\rangle$ is the VEV of the Higgs singlet. The soft-SUSY breaking Lagrangian is also modified according to

$$
\begin{aligned}
-\mathcal{L}_{\text {soft }}= & m_{H_{u}}^{2}\left|H_{u}\right|^{2}+m_{H_{d}}^{2}\left|H_{d}\right|^{2}+m_{S}^{2}|S|^{2} \\
& +\left(\lambda A_{\lambda} H_{u} \cdot H_{d} S+\frac{1}{3} \kappa A_{\kappa} S^{3}+h . c\right)
\end{aligned}
$$

Given $M_{Z}$ and using conditions coming from the minimisation of the Higgs potential, one can choose six independent parameters for the Higgs sector

$$
\lambda, \kappa, A_{\lambda}, A_{\kappa}, \mu_{\mathrm{eff}}, t_{\beta}
$$

where $t_{\beta}=\tan \beta=v_{u} / v_{d}$, the ratio of the two Higgs doublet VEV's: $\left\langle H_{u}^{0}\right\rangle=v_{u},\left\langle H_{d}^{0}\right\rangle=v_{d}$. This is in contrast with the MSSM where two parameters are needed, e.g, $\tan \beta$ and the mass of the Higgs pseudoscalar $M_{A^{0}}$, once the requirement of vanishing tadpoles has been imposed. After EWSB the NMSSM Higgs sector contains three neutral scalar fields, $H_{1}, H_{2}, H_{3}$ and two pseudoscalar neutral ones, $A_{1}, A_{2}$ as well as a charged Higgs $H^{ \pm}$. In the neutralino sector the additional singlino mixes with the bino, wino and Higgsinos fields. The neutralino mass matrix is therefore a $5 \times 5$ one which is diagonalised with a unitary matrix $N$. In the basis $\tilde{\chi}^{0}=\left(-i \tilde{B},-i \tilde{W}_{3}, \tilde{H}_{u}^{0}, \tilde{H}_{d}^{0}, \tilde{S}^{0}\right)$ the neutralino mass matrix reads,

$$
\left(\begin{array}{ccccc}
M_{1} & 0 & -c_{\beta} s_{W} M_{Z} & s_{\beta} s_{W} M_{Z} & 0 \\
0 & M_{2} & c_{\beta} c_{W} M_{Z} & -s_{\beta} c_{W} M_{Z} & 0 \\
-c_{\beta} s_{W} M_{Z} & c_{\beta} c_{W} M_{Z} & 0 & -\mu_{\mathrm{eff}} & -\lambda v_{u} \\
s_{\beta} s_{W} M_{Z} & -s_{\beta} c_{W} M_{Z} & -\mu_{\mathrm{eff}} & 0 & -\lambda v_{d} \\
0 & 0 & -\lambda v_{u} & -\lambda v_{d} & 2 \kappa s
\end{array}\right)
$$

The lightest supersymmetric particle (LSP) can then be expressed as a linear combination of the five gauge eigenstates

$$
\tilde{\chi}_{1}^{0}=N_{11} \tilde{B}+N_{12} \tilde{W}+N_{13} \tilde{H}_{1}+N_{14} \tilde{H}_{2}+N_{15} \tilde{S}
$$

For a pure state the singlino mass is

$$
m_{\tilde{S}}=2 \kappa s
$$

The parameter space of the NMSSM can then be described, in addition to the six Higgs sector parameters, by the same as the MSSM, namely the soft masses for sfermions $M_{\tilde{f}}$, trilinear couplings $A_{f}$ and gaugino masses $M_{1,2,3}$ which are respectively the $\mathrm{U}(1), \mathrm{SU}(2)$ and $\mathrm{SU}(3)$ soft parameters. If the LSP has a dominant singlino component, it can efficiently annihilate through light singlet Higgses as well as light pseudoscalar Higgs singlet $[16,33]$. The MSSM limit is recovered when $\lambda, \kappa \rightarrow 0$ and the equivalent of the mass (squared) of the only physical CP-odd scalar $A^{0}$ of the MSSM is given by

$$
M_{A^{0}}^{2}=\frac{2 \lambda s\left(A_{\lambda}+\kappa s\right)}{\sin 2 \beta}
$$

This is an important proviso when we will compare the NMSSM result with an "equivalent" MSSM spectrum. 


\section{Set-up of the automatic calculation}

One loop processes calculated via the diagrammatic Feynman approach imply the calculation of hundreds of Feynman diagrams and a hand calculation is proned to numerous errors. A high-level of automation is therefore highly desirable, especially if one wants to build a general purpose code. The SloopS code $[25,28,29]$ has been developed in this purpose and applied to astrophysics [25], cosmology [30-32] and also collider physics [29, 34], both in the SM and MSSM. The implementation of the NMSSM has been carried out with LanHEP [35-39] which generates the complete set of NMSSM vertices once the Lagrangian is specified. The calculation of the process $\tilde{\chi}_{1}^{0} \tilde{\chi}_{1}^{0} \rightarrow \gamma Z^{0}$ requires the field renormalisation $\delta Z_{Z \gamma}^{1 / 2}$ which is generated from the (tree-level) $\tilde{\chi}_{i}^{0} \tilde{\chi}_{1}^{0} Z$ vertex through a $Z-\gamma$ one-loop transition. This renormalisation constant is generated in LanHEP by shifting the appropriate fields and defined in the on-shell scheme. The output files are then written in the FormCalc [40,41] conventions which handle the calculation of the cross section. One nice feature of SloopS is the use of a generalised non-linear gauge fixing [42], adapted to the supersymmetric case $[25,28]$, and for this particular work we have extended it to the specific NMSSM Higgs sector. Compared to the MSSM, the gauge-fixing Lagrangian will depend on two additional parameters since the Higgs sector is enlarged with one more scalar and one more pseudoscalar. This allows us to perform a non-trivial check of the gauge independence of the result through the variation of eleven non-linear gauge parameters. The gauge fixing Lagrangian reads in a general form

$$
\mathcal{L}_{G F}=-\frac{1}{\xi_{W}} F^{+} F^{-}-\frac{1}{2 \xi_{Z}}\left|F^{Z}\right|^{2}-\frac{1}{2 \xi_{A}}\left|F^{A}\right|^{2}
$$

where the non-linear functions of the fields $F$ are given by

$$
\begin{aligned}
F^{+}= & \left(\partial_{\mu}-i e \tilde{\alpha} A_{\mu}-i g c_{W} \tilde{\beta} Z_{\mu}\right) W^{\mu+} \\
& \quad+i \xi_{W} \frac{g}{2}\left(v+\tilde{\delta}_{1} H_{1}+\tilde{\delta}_{2} H_{2}+\tilde{\delta}_{3} H_{3}+i\left(\tilde{\kappa} G^{0}+\tilde{\rho}_{1} A_{1}+\tilde{\rho}_{2} A_{2}\right)\right) G^{+} \\
& F^{Z}=\partial_{\mu} Z^{\mu}+\xi_{Z} \frac{g}{2 c_{W}}\left(v+\tilde{\epsilon}_{1} H_{1}+\tilde{\epsilon}_{2} H_{2}+\tilde{\epsilon}_{3} H_{3}\right) G^{0} \\
F^{A}= & \partial_{\mu} A^{\mu}
\end{aligned}
$$

where $G^{0}$ and $G^{ \pm}$are respectively the neutral and charged goldstones. The parameters $\tilde{\alpha}, \tilde{\beta} \cdots \tilde{\epsilon}_{3}$ are generalised gauge fixing parameters.

The ghost Lagrangian $\mathcal{L}^{G h}$ is derived by requiring that the full effective Lagrangian is invariant under BRST transformations. This implies that the full quantum Lagrangian, with $\mathcal{L}_{C}$ and $\mathcal{L}_{G h}$ respectively the classical and ghost Lagrangians,

$$
\mathcal{L}_{Q}=\mathcal{L}_{C}+\mathcal{L}_{G F}+\mathcal{L}_{G h}
$$

be such that $\delta_{\mathrm{BRS}} \mathcal{L}_{Q}=0$ and hence $\delta_{\mathrm{BRS}} \mathcal{L}_{G F}=-\delta_{\mathrm{BRS}} \mathcal{L}_{G h}$ [43]. The BRST transformation for the gauge fields can be found for example in [43]. The NMSSM specific transformations for the scalar fields can be found in the appendix. Within this particular gauge fixing we can 
set $\xi_{W, Z, A}=1$ (avoiding complicated tensor structure for the gauge bosons propagators) and keep the possibility to check the gauge invariance of the result, at the expense of adding new vertices to the model.

The version of LoopTools [44] we used is a modified one which tackles the problem of inverse Gram determinant. This kinematic quantity vanishes when the velocity $v$ is equal to zero. Consequently the reduction algorithm expressing the tensorial loop integrals onto a basis of scalar ones breaks down. The details of this procedure can be found in [25]. In short this method uses the particular kinematics at $v=0$ to reduce, for example, scalar box integrals to a sum of triangle ones. The neutralino mass matrix is diagonalised numerically with a complex unitary matrix $N$ giving positive eigenvalues for the neutralino masses.

\section{Additional NMSSM contributions}

In the MSSM, depending on the nature of the neutralino, the predicted rates for $\langle\sigma v\rangle_{\gamma \gamma / Z}$ can be quite different. The highest ones are reached when the LSP is mostly wino or higgsino like. In the former case the dominant contributions are from loops with gauge bosons, since in the wino case the most important coupling is $\tilde{\chi}_{1}^{0} \tilde{\chi}_{1}^{ \pm} W^{\mp}$. For a Higgsino $\tilde{\chi}_{1}^{0}$ the dominant contribution would be vertex corrections through a $Z^{0}$ boson in the s-channel as the $\tilde{\chi}_{1}^{0} \tilde{\chi}_{1}^{0} Z$ coupling is proportional to

$$
g_{\tilde{\chi}_{1}^{0} \tilde{\chi}_{1}^{0} Z} \propto N_{13}^{2}-N_{14}^{2}
$$

The third possible case, a bino-like neutralino, would annihilate mainly through box diagrams containing right-handed sfermions since the bino, as the superpartner of the $B$ boson, couples to sparticles possessing the highest hypercharge. A bino like LSP gives generically lower rates than the two other cases as its couplings to other particles are weaker. Therefore if one wants to stick to a mostly bino neutralino while giving good prospects for the spectral photon lines, significant mixing with the other components is needed.

In going from the MSSM calculation to the NMSSM one, two major differences appear: on the one hand the NMSSM couplings now depend on the parameters $\lambda$ and $\kappa$, and on the other hand new diagrams are to be computed. Concerning the case of the modified couplings, if the NMSSM neutralino is a mostly bino, wino or higgsino, we do not expect major differences with the MSSM, as long as the singlino component is negligible. As the neutralino is a Majorana fermion, at vanishing relative velocity the LSP pair carries pseudoscalar quantum numbers. Therefore the NMSSM additional diagrams are the ones with pseudoscalar $A_{1}, A_{2}$ s-channel exchange. The relevant diagrams are depicted in figure 1. Note that the diagram with the chargino loop should be understood as containing both "flavours" of charginos since the couplings $A_{k} \tilde{\chi}_{i}^{ \pm} \tilde{\chi}_{j}^{\mp}$ are non-diagonal. Note also that the couplings $A_{k} \tilde{f}_{i} \tilde{f}_{j}$ are also non-diagonal in family space because we stick to real soft SUSY breaking terms. Hence one could think that the NMSSM brings also new contributions containing loops with different flavours of sfermions. In fact their amplitudes cancel when summing over all the flavours. ${ }^{2}$ Furthermore due to our particular gauge-fixing Lagrangian

\footnotetext{
${ }^{2}$ This is of course also the case in the MSSM.
} 

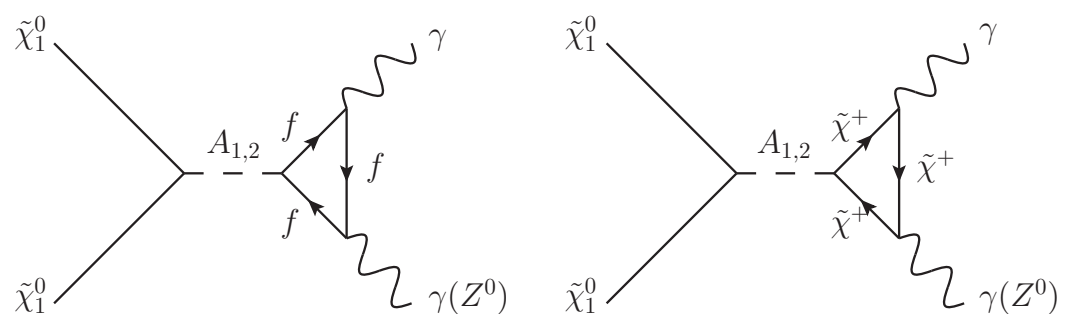

Figure 1. Additional NMSSM diagrams with s-channel pseudoscalar exchange for the processes $\tilde{\chi}_{1}^{0} \tilde{\chi}_{1}^{0} \rightarrow \gamma \gamma\left(Z^{0}\right)$. The label $f$ stands for a SM fermion and $\chi^{+}$to a chargino.

in eq. (2.1), we generate couplings of the pseudoscalars to the charged goldstones $G^{ \pm}$and to the charged ghosts $c^{W}, \bar{c}^{W}$, proportional to $\tilde{\alpha}$ and $\tilde{\rho}_{1,2}$. These additional couplings are not present in the linear gauge. However, just like the case of the sfermions coupling to $A_{1,2}$ the related amplitudes vanish identically. This is however not surprising since there exists no "tree-level" coupling of the pseudoscalars to a pair of gauge bosons. In turn, the process with $\gamma \gamma$ will involve only the non-linear gauge parameter $\tilde{\alpha}$ and the process with $\gamma Z^{0}$ the parameter $\tilde{\beta}$ in addition. Obviously the final result should not depend on these two parameters, they are used as a check on the gauge invariance of the cross sections, which we ascertained.

The use of the non-linear gauge fixing Lagrangian of eq. (2.1) in the 't Hooft-Feynman gauge $\left(\xi_{A}=\xi_{Z}=\xi_{W}=1\right)$ also enables us to cancel, for example, the $W^{+} G^{-} \gamma$ vertex, by setting $\tilde{\alpha}=-1$, which cancels a corresponding piece of the original trilinear sector of the Lagrangian, leading to a vanishing total $W^{+} G^{-} \gamma$ trilinear coupling (see [45] and references therein). We can perform the same trick for the coupling $W^{+} G^{-} Z^{0}$ by setting $\tilde{\beta}$ to an appropriate value. Finally, as a further check on the implementation we took the input parameters for the six scenarios presented in [25], compared with our results by taking the MSSM limit, and found a perfect agreement.

The FERMI collaboration has already provided their limits [17] on the $\gamma \gamma$ and $\gamma Z^{0}$ channels for different photon energies $E_{\gamma}$, hence we did not model the propagation of the photon signal and we restricted ourselves to the discussion of $\langle\sigma v\rangle_{\gamma \gamma}$ and $\langle\sigma v\rangle_{\gamma Z}$.

\section{Benchmark points and comparison with the MSSM}

As a first application of our code we computed the gamma-ray lines for the SUGRA and GMSB benchmark points from [26, 27] and NMSSMTools_2.3.5 [46-48]. For a discussion about these benchmarks we refer to their respective publication $[26,27]$. We then compared with the present limit given by FERMI [17] and against the MSSM calculation, performed with the SloopS code with a MSSM model file, like in [25], to see how much precision one would need to distinguish them. The input parameters for the MSSM parameters are the same as in the NMSSM taken in the decoupling limit $\lambda, \kappa \rightarrow 0$ and using eq. (1.8). It is important to note that, in order to preserve gauge invariance, we only took the input parameters obtained after the RGE running (therefore $\overline{\mathrm{DR}}$ parameters) to the low-energy 


\begin{tabular}{|c|c|c|c|c|}
\hline Parameter & SUGRA-P1 & SUGRA-P2 & SUGRA-P3 & SUGRA-P4 \\
\hline$M_{1}$ & 211.62 & 211.62 & 211.86 & 333.80 \\
\hline$M_{2}$ & 391.86 & 391.86 & 392.51 & 613.91 \\
\hline$\mu$ & 968.62 & 968.62 & 938.41 & -206.17 \\
\hline$t_{\beta}$ & 10.000 & 10.000 & 10.000 & 3.0413 \\
\hline$M_{\tilde{l}_{1,2}^{R}}$ & 257.51 & 257.51 & 256.38 & 702.15 \\
\hline$M_{\tilde{l}_{3}^{R}}$ & 221.13 & 221.13 & 221.21 & 698.94 \\
\hline$M_{\tilde{u}_{1,2}^{R}}$ & 986.12 & 986.12 & 985.64 & 1674.5 \\
\hline$M_{\tilde{u}_{3}^{R}}$ & 565.23 & 565.23 & 590.90 & 527.83 \\
\hline$M_{\tilde{d}_{1,2}^{R}}$ & 981.60 & 981.60 & 981.17 & 1609.0 \\
\hline$M_{\tilde{d}_{3}^{R}}$ & 966.15 & 966.15 & 966.20 & 1606.6 \\
\hline$A_{\mu}$ & -1777.5 & -1777.5 & -1698.7 & -2531.2 \\
\hline$A_{\tau}$ & -1763.0 & -1763.0 & -1682.8 & -2524.8 \\
\hline$A_{b}$ & -2611.8 & -2611.8 & -2535.2 & -3730.5 \\
\hline$A_{t}$ & -1431.5 & -1431.5 & -1362.6 & -1765.8 \\
\hline \multicolumn{5}{|c|}{ MSSM specific parameter } \\
\hline$M_{A^{0}}$ & 943.65 & 943.65 & 935.19 & 702.11 \\
\hline \multicolumn{5}{|c|}{ NMSSM specific parameters } \\
\hline$\lambda$ & 0.1 & 0.1 & 0.4 & 0.49 \\
\hline$\kappa$ & 0.1089 & 0.1089 & 0.3037 & 0.0554 \\
\hline$A_{\lambda}$ & -963.91 & -963.91 & -620.23 & -684.51 \\
\hline$A_{\kappa}$ & -1.5893 & -1.0934 & -11.158 & 151.73 \\
\hline
\end{tabular}

Table 1. SUSY input parameters for the SUGRA benchmarks. Masses are in GeV.

scale and considered them as physical parameters, no mass correction or effective couplings have been considered. The self-annihilation of the neutralino is mainly driven by its mass and composition, so the first question to ask when comparing the MSSM with the NMSSM is whether their mass and composition are similar.

\subsection{SUGRA}

The SUGRA input parameters are taken from the version 2.3.5 of NMSSMTools as they have been substantially modified since the publication of [26]. The most relevant parameters are given in table 1 . The first three scenarios (P1, P2, P3) exhibit a mostly bino-like neutralino and are quite similar, as far as only the neutralino/chargino sector is concerned. In the four cases the lightest neutralino is heavy enough $\left(m_{\tilde{\chi}_{1}^{0}} \simeq 211 \mathrm{GeV}\right)$ such that the $\gamma Z^{0}$ final state is open. As the first three benchmark scenarios give a bino-like LSP, we can already anticipate that the NMSSM and MSSM will give similar results as they have a very similar composition. However we expect that the fourth scenario P4 will give a clearly distinctive signature since in this case the lightest NMSSM neutralino $\tilde{\chi}_{1}^{0}$ is $99 \%$ singlino with mass $m_{\tilde{\chi}_{1}^{0}} \simeq 60 \mathrm{GeV}$. The final results can be found in table 2 . 


\begin{tabular}{|c|c|c|c|}
\hline Model & $m_{\chi}(\mathrm{GeV})$ & $\langle\sigma v\rangle_{\gamma \gamma} \times 10^{30}\left[\mathrm{~cm}^{3} \mathrm{~s}^{-1}\right]$ & $\langle\sigma v\rangle_{\gamma Z} \times 10^{30}\left[\mathrm{~cm}^{3} \mathrm{~s}^{-1}\right]$ \\
\hline \multicolumn{3}{|c|}{ SUGRA-P1/P2 } \\
\hline NMSSM & 210.74 & 3.9840 & 1.7391 \\
MSSM & 210.74 & 3.9836 & 1.7393 \\
\hline \multicolumn{3}{|c|}{ SUGRA-P3 } \\
\hline NMSSM & 210.92 & 4.0103 & 1.7326 \\
MSSM & 210.93 & 4.0074 & 1.7348 \\
\hline \multicolumn{3}{|c|}{ SUGRA-P4 } \\
\hline NMSSM & 59.963 & $2.715710^{-3}$ & $2.101210^{-3}$ \\
MSSM & 200.43 & 61.866 & 205.28 \\
\hline
\end{tabular}

Table 2. Rates for the loop-induced annihilation of two neutralinos into $\gamma \gamma / Z^{0}$ for the SUGRA benchmarks.

We see that, unless the NMSSM neutralino has a radically different composition from the MSSM case, these two frameworks are almost indistinguishable. Needless to say that this is also a good indication of the implementation of the NMSSM model since in the decoupling limit (which is the case for these two scenarios) we should recover the MSSM. The benchmarks SUGRA-P1 and SUGRA-P2 give equal results because they only differ by the value of $A_{\kappa}$, which has an impact only on the mass of the lightest pseudoscalar, which is not relevant in these cases. A difference is clearly visible when the NMSSM neutralino is mostly singlino, see the SUGRA-P4 case, whereas the MSSM neutralino is higgsino-like. Obviously for this latter case the comparison is not very meaningful from an experimental point of view since the inputs would be the energy of the photon $E_{\gamma}$ and the rate $\langle\sigma v\rangle$, which are clearly different in this benchmark point. Considering only the first three NMSSM SUGRA scenarios, the gamma-line observable does not permit us to discriminate them. The distinct features of the NMSSM Higgs sector are quite decoupled from the rest because $\lambda$ and $\kappa$ are quite small for the first two scenarios. The situation is different for the third scenario where $\lambda$ and $\kappa$ take higher values and the NMSSM Higgs sector exhibits a different spectrum than the MSSM one because, in this case, the singlet component mixes notably with the doublets. Nevertheless, we recall that as the neutralino is a Majorana particle, it forms a pseudoscalar state such that its couplings to scalar Higgses are suppressed at vanishing relative velocities, and therefore the LSP is mainly sensitive to the pseudoscalar part of the Higgs spectrum. Moreover the singlino-like neutralino is very heavy compared to the rest of the neutralino spectra, such that it does not bring significant mixing. Thus the couplings of the lightest neutralino does not depart much with respect to the MSSM case. In addition a MSSM bino-like neutralino does not couple much to Higgs at low $t_{\beta},{ }^{3}$ such that in the three bino scenarios considered the annihilations are driven by box diagrams with sfermions, in particular staus. Let us now comment the SUGRA-P4 scenario. The NMSSM benchmark point gives a LSP which is almost a pure singlino and couples very feebly to other particles. Hence we obtain a very small rate, compared to the

\footnotetext{
${ }^{3}$ The input value of $t_{\beta}$ is equal to 10 at the $M_{Z}$ scale
} 


\begin{tabular}{|c|c|c|c|c|c|}
\hline Parameter & GMSB-P1 & GMSB-P2 & GMSB-P3 & GMSB-P4 & GMSB-P5 \\
\hline$M_{1}$ & 473.32 & 473.42 & 136.69 & 136.91 & 497.49 \\
$M_{2}$ & 860.25 & 859.50 & 257.30 & 258.02 & 905.29 \\
$\mu$ & 1391.6 & 233.89 & 660.05 & 554.37 & 1363.0 \\
$t_{\beta}$ & 8.4821 & 1.6277 & 1.5982 & 1.9000 & 50.055 \\
$M_{\tilde{l}_{1,2}^{R}}$ & 692.31 & 689.42 & 138.22 & 133.34 & 622.57 \\
$M_{\tilde{l}_{3}^{R}}$ & 686.00 & 689.20 & 138.17 & 133.28 & 429.74 \\
$M_{\tilde{u}_{1,2}^{R}}$ & 231.81 & 231.70 & 771.61 & 777.47 & 2439.5 \\
$M_{\tilde{u}_{3}^{R}}$ & 187.05 & 176.49 & 631.97 & 660.97 & 20361.8 \\
$M_{\tilde{d}_{1,2}^{R}}$ & 228.40 & 228.27 & 767.45 & 773.63 & 2413.4 \\
$M_{\tilde{d}_{3}^{R}}$ & 227.61 & 228.23 & 767.36 & 773.52 & 2238.0 \\
$A_{\mu}$ & -424.24 & -321.01 & -54.511 & -46.704 & -208.15 \\
$A_{\tau}$ & -423.01 & -318.15 & -54.333 & -46.573 & -184.48 \\
$A_{b}$ & -2115.1 & -1990.8 & -439.67 & -398.57 & -166.66 \\
$A_{t}$ & -1558.5 & -1265.4 & -328.32 & -314.23 & -143.41 \\
\hline \multicolumn{5}{|c|}{ MSSM specific parameter } \\
\hline$M_{A^{0}}$ & 1756.1 & 2884.8 & 875.97 & 714.730 & 1061.3 \\
\hline \multicolumn{5}{|c|}{ NMSSM specific parameters } \\
\hline$\lambda$ & 0.002 & 0.5 & 0.6 & 0.6 & 0.01 \\
$\kappa$ & 0.0045 & 0.4351 & 0.4540 & 0.3969 & -0.0007 \\
$A_{\lambda}$ & -56.263 & -449.46 & 23.012 & 13.083 & 114.94 \\
$A_{\kappa}$ & -158.89 & -2278.7 & 0.0315 & 0.7354 & 0.0048 \\
\hline
\end{tabular}

Table 3. SUSY input parameters for the GMSB benchmarks. Masses are in GeV.

Higgsino-like LSP in the P4 MSSM benchmark, since in this case the pair annihilation is quite efficient as the LSP has $\mathrm{SU}(2)$ quantum numbers.

All in all, taking into account the latest limits provided by FERMI [17], none of these benchmarks points are excluded and far away from FERMI sensitivity. As these benchmarks are representative of the NMSSM phenomenology this means and confirms the FERMI collaboration statement that the present limits on the gamma-ray line are too weak compared to the typical cross sections for a conventional thermal NMSSM LSP. We also remark that the situation is worse for a mostly singlino neutralino. This is quite generic for a singlino $\tilde{\chi}_{1}^{0}$ unless some specific mechanism is at play, we will come back to this point later. As a final comment to this section we also observe that the $\gamma Z$ mode gives generically smaller rates (in both the MSSM and NMSSM) than the $\gamma \gamma$ one, as usually claimed, except for the SUGRA-P4 MSSM scenario.

\subsection{GMSB}

We now turn to the GMSB benchmark points. These were taken once again from NMSSMTools_2.3.5 package [46-48]. The most relevant ones are depicted in table 3 . The 


\begin{tabular}{|c|c|c|c|}
\hline Model & $m_{\chi}(\mathrm{GeV})$ & $\langle\sigma v\rangle_{\gamma \gamma} \times 10^{30}\left[\mathrm{~cm}^{3} \mathrm{~s}^{-1}\right]$ & $\langle\sigma v\rangle_{\gamma Z} \times 10^{30}\left[\mathrm{~cm}^{3} \mathrm{~s}^{-1}\right]$ \\
\hline \multicolumn{3}{|c|}{ GMSB-P1 } \\
\hline NMSSM & 472.42 & 0.2915 & 0.1176 \\
MSSM & 472.42 & 0.2915 & 0.1176 \\
\hline \multicolumn{3}{|c|}{ GMSB-P2 } \\
\hline NMSSM & 472.47 & 0.2981 & 0.1185 \\
MSSM & 472.47 & 0.2981 & 0.1185 \\
\hline \multicolumn{3}{|c|}{ GMSB-P3 } \\
\hline NMSSM & 133.01 & 12.862 & 6.0282 \\
MSSM & 133.02 & 12.827 & 6.0466 \\
\hline \multicolumn{3}{|c|}{ GMSB-P4 } \\
\hline NMSSM & 132.50 & 14.490 & 7.1262 \\
MSSM & 132.55 & 14.460 & 7.1544 \\
\hline \multicolumn{3}{|c|}{ GMSB-P5 } \\
\hline NMSSM & 196.84 & $2.626410^{-10}$ & $5.559610^{-11}$ \\
MSSM & 496.83 & 1.0884 & 0.5055 \\
\hline
\end{tabular}

Table 4. Rates for the loop-induced annihilation of two neutralinos into $\gamma \gamma / Z^{0}$ for the GMSB benchmarks.

original GMSB phenomenology of the NMSSM has been studied in [27]. The spectral lines results can be found in table 4 . The GMSB-P1 and P2 benchmarks give both almost similar results with $m_{\tilde{\chi}_{1}^{0}} \simeq 472 \mathrm{GeV}$. We observe no major discrepancy between the NMSSM and MSSM results, even in scenario P2 where the NMSSM sector is not in the decoupling regime since $\lambda$ and $\kappa$ take quite high values. Nevertheless it still has no impact because the LSP is $99 \%$ bino-like and has MSSM-like couplings. Note that for these two points the LSP mass is outside the energy range published in [17, 49]. For benchmarks P3 and P4 the differences between the NMSSM and the MSSM are tiny, already at the level of the mass of the lightest neutralino $m_{\tilde{\chi}_{1}^{0}}$, in turn the rates are slightly different. This is mostly due to the fact that the signal is computed at a different center of mass energy. Even though it would correspond to different signal/energy bins, we can anyway compare them since the mass difference is quite small and require a high level of precision. Therefore even discriminating between the two models is also extremely challenging, both in $\gamma \gamma$ and $\gamma Z^{0}$ final states. As well as the mSUGRA benchmark points where the LSP is mostly bino-like, the annihilation is driven by box diagrams involving right-handed sfermions, and in particular the right-handed sleptons since they possess the lower masses of the sfermion spectrum. The benchmarks P3 and P4 give an enhanced rate because the right-handed slepton masses are close to the mass of the neutralino. In addition the bino-like neutralino in the P4 scenario has a substantial higgsino component, increasing its couplings to the $Z^{0}$ boson. Finally the scenario P5 gives an extremely tiny signal where the LSP is mostly singlino and the s-channel exchange of the singlet pseudoscalar is extremely suppressed since its mass is around $1 \mathrm{GeV}$. 


\section{Spectral lines from light neutralino annihilation}

We have seen in the previous section that a mostly bino LSP in the NMSSM mimics the MSSM result and gives quite low predictions with respect to the present experimental limits. We propose in this section to investigate how the signal can be improved in the NMSSM, with features distinct from the MSSM. Nevertheless, let us recall how we can increase the gamma-line signal with features similar to the MSSM. In a generic neutralino case, one can look for a point in parameter space where a $Z^{0}$ resonance is hit, with $m_{\tilde{\chi}_{1}^{0}} \simeq$ $M_{Z} / 2 \simeq 45 \mathrm{GeV}$, not forgetting the fact that the $\gamma Z^{0}$ channel would require special care since we would produce the $Z^{0}$ boson at threshold. Second a mostly wino, higgsino or a mixture of a bino with one of these states would result in a signal with better prospects, like in the MSSM. However such type of neutralinos must be quite heavy, especially for wino- or Higgsino-like neutralinos. Indeed, if one wants to reproduce the correct CDM abundance, typically the masses must be of order the TeV scale. ${ }^{4}$ Motivated by results indicative of light dark matter, we focused on the case of a NMSSM light neutralino, which can have very different properties than the one of the MSSM. It is very difficult in the MSSM to have a very light neutralino which do not overclose the Universe and at the same time fulfills the collider constraints $[7,8,13,14]$. Reconciliating these two criteria can be provided by the NMSSM more easily. Indeed the lightest of the two CP-odd Higgs bosons can be much lighter than the single CP-odd Higgs boson of the MSSM without violating collider constraints. Another feature that cannot be reproduced in the MSSM is obviously the one where the LSP is mostly singlino. We have seen that on general grounds the signal is very low for such a $\tilde{\chi}_{1}^{0}$. Nevertheless, just like with a $Z^{0}$ boson we can enhance the signal by hitting a resonance, this time with one pseudoscalar Higgs. This mechanism has already been pointed out in [16], and is also invoked to give the right relic density when the LSP possesses an important singlino component [7, 33]. We therefore investigated to which extent the signal can be enhanced for such a mechanism with neutralinos lighter than $15 \mathrm{GeV}$, and if the present limits can be used to further constrain the parameter space found in [7]. Such light neutralinos would evade the present constraints from the published FERMI data on gamma-lines since their threshold is around $30 \mathrm{GeV}$ [17]. However Vertongen and Weniger [49] have derived a broader energy range which starts from $1 \mathrm{GeV}$ and extends up to $300 \mathrm{GeV}$, see table. 3 of [49]. As a consequence we will only discuss how to increase the $\gamma \gamma$ signal since the $\gamma Z^{0}$ channel is closed in this range, and we will compare our predicted rates with the limits given in [49].

\subsection{Light singlet pseudoscalar Higgs resonance}

Light neutralinos in the NMSSM have been studied for example in [7, 51-53]. A singlinolike $\tilde{\chi}_{1}^{0}$ emerges when $\kappa \ll 1$ and to ensure at the same time a light singlet pseudoscalar the parameter $A_{\kappa}$ should be small either (in the decoupling limit $m_{A_{1}}^{2} \simeq-3 \kappa A_{\kappa} s$ ). Light singlino-like LSP are found mainly in two regions of parameter space: one at small $\lambda$ with very small $|\kappa|$, and another at large $\lambda$ with slightly larger $|\kappa|$ allowed, see [51]. To

\footnotetext{
${ }^{4}$ Scenarios with heavy MSSM wino-like or Higgsino-like LSP also require special care for the gamma-line computation, in particular the treatment of the Sommerfeld singularity, see the pioneer work of [50]
} 
hit a light singlet pseudoscalar Higgs resonance we obviously need $m_{\tilde{\chi}_{1}^{0}} \simeq m_{A_{1}} / 2$. This can be achieved with a pure singlino-like neutralino but, in practice, neutralinos fulfilling this criteria and accounting for the present cosmic abundance of dark matter measured by WMAP are mixed states, like a bino-singlino or bino-higgsino like neutralino. In any case the neutralino obviously needs some substantial component related to the Higgs sector to couple to the pseudoscalars. Such light pseudoscalars and neutralinos can be motivated in models were an approximate U(1) R-symmetry or U(1) Peccei-Quinn symmetry holds (see [51] and references therein) and therefore their "lightness" is more "natural" than in the MSSM. To see in which extent the signal can be boosted by this mechanism we performed a scan over the parameter spaced spanned by the 11 parameters

$$
M_{1}, M_{2}, \mu, t_{\beta}, \lambda, \kappa, A_{\lambda}, A_{\kappa}, A_{t}, m_{\tilde{l}}, m_{\tilde{q}}
$$

as in [7] and computed the rate $\langle\sigma v\rangle_{\chi \chi \rightarrow \gamma \gamma}$ as only this channel is open. The parameters $m_{\tilde{l}}$ and $m_{\tilde{q}}$ are common masses to the sleptons and squarks respectively and are taken in the same range as in [7]. However to find these points we did not apply any constraints, we just tried to find points in parameter space which where minimizing the difference

$$
\Delta M=\frac{2 m_{\tilde{\chi}_{1}^{0}}-m_{A_{1}}}{m_{A_{1}}}
$$

such that we observed an enhanced signal, and with $m_{\tilde{\chi}_{1}^{0}} \leq 15.5 \mathrm{GeV}$. No width to the pseudoscalar $A_{1}$ propagator was introduced during the calculation, since we did not encounter any numerical problems. This is due to the fact that the width of the pseudoscalar is extremely narrow (numerically $10^{-7} \lesssim \Gamma_{A_{1}} \lesssim 10^{-4}$ ). Anyway, as a check, we added a width to the propagator of the lightest pseudoscalar for the points giving the highest rates, i.e, very close to the resonance, and we observed a maximum decrease of $\sim 20 \%$ of the signal, so these points would still be excluded. As expected, the effect of the width was less and less important (reaching the percent level) the more we were going away from the resonance, where more points are allowed. The effects of the width for these allowed points is therefore lower than the present experimental accuracy. The result of this scan is displayed in figure 2. Red squared points are scenarios where the LSP is more than $90 \%$ bino, blue diamond-shaped points more than $90 \%$ singlino and the green stars are a mixture of both. We displayed only the region where $|\Delta M| \leq 1 \%$. We can see that for this mechanism to boost significantly the signal a high degree of fine-tuning of $\Delta M$ is required. Very few bino-like neutralinos scenarios were found and give a signal lower than $10^{-28} \mathrm{~cm}^{3} \mathrm{~s}^{-1}$, showing that this mechanism is specific to the NMSSM. Most of the points possess also a significant Higgsino component which ranges from the percent level to approximately $15 \%$. Notice also that as soon as $|\Delta M| \gtrsim 0.2 \%$ the rate significantly decreases and more points were produced with $m_{A_{1}} \geq 2 m_{\tilde{\chi}_{1}^{0}}$. We observe that in the most extreme case we can reach a signal as large as $10^{-25} \mathrm{~cm}^{3} \mathrm{~s}^{-1}$, for a mixed $\tilde{\chi}_{1}^{0}$, which is $88 \%$ singlino and $9 \%$ Higgsino while $\Delta M \simeq 0.001 \%$. However recall that we did not apply any constraining criteria for this scan. In figure 3 we display the result of this scan with respect to the limits given in [49]. This time we picked up points with $|\Delta M| \leq 15 \%$, this selected the mass window $4 \leq m_{\tilde{\chi}_{1}^{0}} \leq 15.5 \mathrm{GeV}$. We see that the highly fine-tuned points (with 


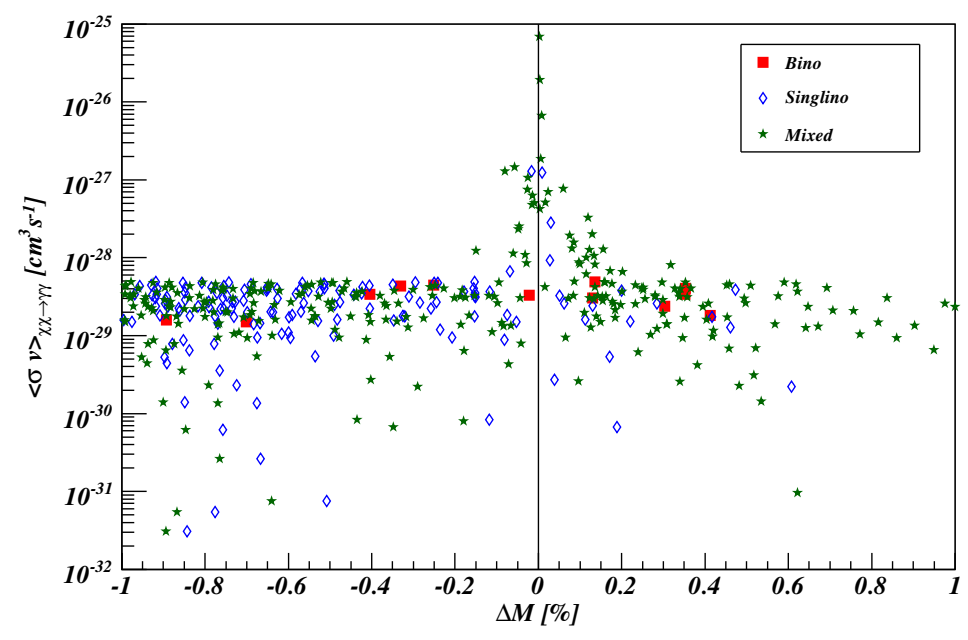

Figure 2. Gamma-ray line rate with respect to the mass difference $\Delta M=\left(2 m_{\tilde{\chi}_{1}^{0}}-m_{A_{1}}\right) / m_{A_{1}}$ for several composition of the neutralino (see text for details).

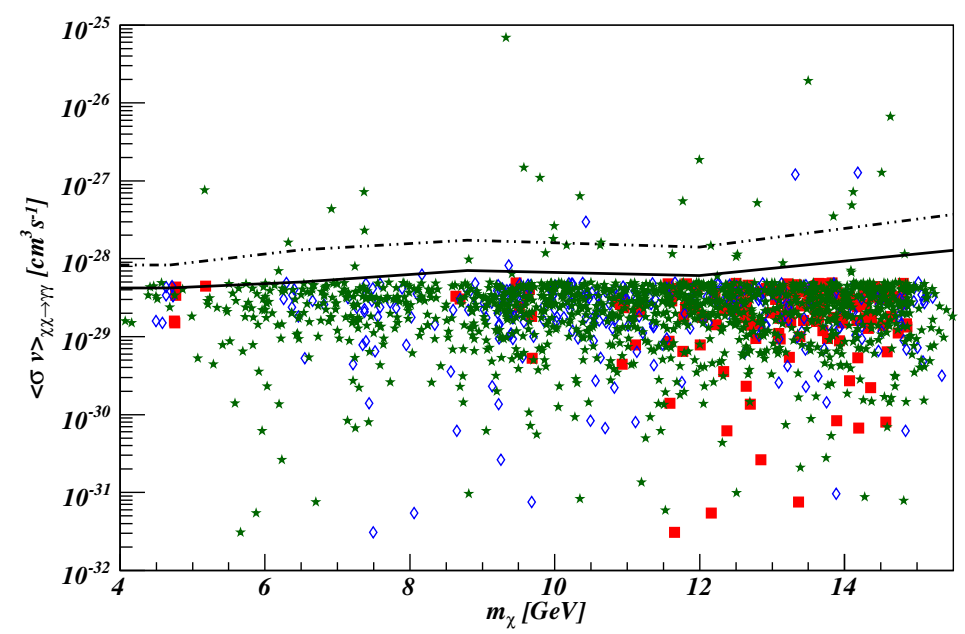

Figure 3. Gamma-ray line rate with respect to $m_{\chi}$. The limits of [49] are drawn for the halo (dashed dotted line) and the galactic center (solid line). The conventions for the neutralino composition follow those of figure 2 .

$|\Delta M| \leq 0.2 \%$ and $\langle\sigma v\rangle_{\gamma \gamma} \gtrsim 10^{-28} \mathrm{~cm}^{3} / \mathrm{s}$ ) would be excluded by the spectral lines searches. However we found that most of the points are just below the FERMI sensitivity, especially concerning the galactic center observation. Therefore an increase by at least one order of magnitude would much more constrain the s-channel pseudoscalar resonance, if no signal is reported. It is also worth to note that the highly fine-tuned scenarios tend also to give a very low relic density ${ }^{5}$ hence giving up the possibility of the LSP to account for the actual amount of dark matter. Moreover, as we will discuss in the next section, the gamma-rays from dwarf spheroidal galaxies (dSph) $[54,55]$ give more powerful constraints.

\footnotetext{
${ }^{5}$ Nevertheless we found some points satisfying this criteria.
} 


\subsection{Gamma-ray lines as a further constraint on the NMSSM parameter space.}

Interestingly the studies led by $[7,56]$ found that light NMSSM neutralinos that are compatible with CoGeNT and satisfy all the constraints they applied (colliders, relic density of DM, flavour observables) are accompanied by a light pseudoscalar and/or scalar singlet. Generally the aforementioned studies showed that the highest elastic scattering cross-sections are reached for low values of $m_{H_{1}}$ and can satisfy the WMAP constraint when $m_{H_{1}} \simeq 2 m_{\tilde{\chi}_{1}^{0}}$, because in this case the pair annihilation of neutralinos is enhanced by a s-channel resonance. Obviously the gamma-ray lines cannot constrain such scenarios as this channel is suppressed for low-velocities. Scenarios that could be constrained are therefore rather those where $m_{\tilde{\chi}_{1}^{0}} \simeq m_{A_{1}} / 2$.

The authors of [54] computed the secondary gamma rays produced in dSph from the pair annihilation of DM particles into quarks and/or taus which subsequently hadronise and decays into pions, finally decaying into photons. The authors then compared their findings with the FERMI $95 \%$ limits on gamma-ray emission from dSph [57]. The authors provided us with 14 points of their MCMC scan giving a large pair annihilation cross section but safe with respect to dSph limits and direct detection searches. The points are sampled in each bins of $m_{\tilde{\chi}_{1}^{0}}$ between 1 and $15 \mathrm{GeV}$. We then used these input parameters to evaluate the rate of the gamma-lines to see if the corresponding limits derived in [49] from the FERMI data on spectral lines [17] could further constrain these scenarios. The mass spectrum of these parameter points fall into the range where $m_{A_{1}}$ is not far from $2 m_{\tilde{\chi}_{1}^{0}}$. However models for which the mass difference between the LSP and the pseudoscalar is highly fine-tuned are already excluded by the Draco limits [54]. We therefore do not expect very bright signals. We collected in table 5 the total annihilation cross section of neutralinos into quarks and/or taus, taken from [54], with our results concerning the annihilation into two photons, since here again the range of $m_{\tilde{\chi}_{1}^{0}}$ studied is too low for the production of the $\gamma Z^{0}$ final state. As can be seen from table 5, the gamma-line rate is at most four orders of magnitude lower than the rate coming from other indirect search channels. Of course since the direct annihilation into photons is loop-suppressed we cannot expect that it can significantly contribute to the total annihilation cross section producing gamma rays. For the computation of gamma ray fluxes emerging from dark matter annihilation in dwarf spheroidal galaxies, the "direct" annihilation into photons can then safely be neglected. If we now compare the results in table 5 with the limits of [49], which are at best of order $10^{-29} \mathrm{~cm}^{3} / \mathrm{s}$ for a very light neutralino (less than approximately $5 \mathrm{GeV}$ ), none of these points is further constrained. Most of the models exhibits a spectrum with a mass difference $\Delta M$ too high to enhance significantly the signal, and relatively low mass sleptons. The point with $m_{\tilde{\chi}_{1}^{0}}=14.48 \mathrm{GeV}$ gives the best signal because $\Delta M \simeq 0.5 \%$ with a LSP at $92 \%$ singlino and $8 \%$ Higgsino. A sensitivity increased by at least two order of magnitude would be needed to exclude this point with respect to the constraints from gamma-lines searches. In consequence we conclude that the present limits on gamma ray lines can constrain models with a very low mass difference $\Delta M$ but they are not competing with the gamma ray searches from dwarf spheroidal galaxies anyway. This can be easily understood by the fact that photons produced from processes like $\tilde{\chi}_{1}^{0} \tilde{\chi}_{1}^{0} \rightarrow q \bar{q} / \tau \bar{\tau}$ are leading-order dominated and experiments have not yet reached a sensitivity down to loop effects, like the spectral lines. 


\begin{tabular}{|c|c|c|}
\hline $\begin{array}{c}m_{\tilde{\chi}_{1}^{0}} \\
{[\mathrm{GeV}]}\end{array}$ & $\begin{array}{c}\langle\sigma v\rangle_{\chi \bar{\chi} \rightarrow q \bar{q}, \tau \bar{\tau}} \times 10^{27} \\
{\left[\mathrm{~cm}^{3} \mathrm{~s}^{-1}\right]}\end{array}$ & $\begin{array}{c}\langle\sigma v\rangle_{\gamma \gamma} \times 10^{30} \\
{\left[\mathrm{~cm}^{3} \mathrm{~s}^{-1}\right]}\end{array}$ \\
\hline 0.976 & 0.209 & 0.00008 \\
\hline 2.409 & 0.297 & 0.00267 \\
\hline 3.342 & 0.345 & 0.00345 \\
\hline 4.885 & 3.298 & 0.00262 \\
\hline 5.626 & 5.389 & 0.00410 \\
\hline 6.551 & 3.547 & 0.00427 \\
\hline 7.101 & 2.425 & 0.00664 \\
\hline 8.513 & 2.161 & 0.00220 \\
\hline 9.274 & 2.497 & 0.00655 \\
\hline 10.27 & 2.323 & 0.01881 \\
\hline 11.50 & 2.575 & 0.02456 \\
\hline 12.74 & 3.224 & 0.02003 \\
\hline 13.51 & 9.571 & 0.17487 \\
\hline 14.48 & 148.4 & 2.87500 \\
\hline
\end{tabular}

Table 5. Comparison between dark matter annihilation into quarks and/or taus (values are taken from [7]) and the loop-induced one into photons in the NMSSM for each of the bins between 1 and $14 \mathrm{GeV}$.

Conclusion. The mono-energetic gamma ray line signal has spectacular features: a clear "smoking-gun" signature and a direct relation to the mass of the dark matter particle. Moreover it do not suffer from astrophysical uncertainties and depend only on the assumption of the dark matter halo. However discriminating it from the overwhelming astrophysical background (supernovæ, pulsars, cosmic-rays...) is experimentally extremely challenging and requires a fine energy resolution. Furthermore the inclusive channels $\chi \chi \rightarrow q \bar{q}(W W, Z Z)$ produce a featureless continuous spectrum of gammas coming from the decay or hadronisation of the final state, only cut-off at a maximum energy corresponding to the mass of the DM particle. This contribution is stronger than the monochromatic photons, since it is a tree-level dominated process. In this paper we evaluated numerically in the NMSSM framework the process $\tilde{\chi}_{1}^{0} \tilde{\chi}_{1}^{0} \rightarrow \gamma \gamma$ and provided for the first time result for $\tilde{\chi}_{1}^{0} \tilde{\chi}_{1}^{0} \rightarrow \gamma Z^{0}$. This is the first implementation of the NMSSM framework in SloopS and paves the way for a future renormalisation of each sector, like what was done for the $\operatorname{MSSM}[28,29]$. We performed the calculation at $v=0$ and checked the result with respect to gauge invariance thanks to an NMSSM extension of the non-linear gauge-fixing used in [28]. As a further check we compared the NMSSM result in the "MSSM-limit" and the pure MSSM one and found an excellent agreement. As a first application we computed the $\gamma \gamma\left(Z^{0}\right)$ signal for several benchmarks found in the literature [26, 27]. We compared with an "equivalent" MSSM spectrum and the limits given by FERMI [17] and in [49]. We found that unless the lightest neutralino has a significant singlino component, the two models produce very similar rates and discriminating between them requires a high level of experimental 
precision. This is particular true if in both cases the LSP is bino-like. However, if the neutralino is to be the dark matter candidate and light, discriminating the NMSSM from the MSSM should be easier since light neutralinos and Higgs scalars are more "natural" in the NMSSM, thanks to an approximate Peccei-Quinn symmetry. A low mass WIMP is therefore easier to accommodate in the NMSSM than the MSSM. In the NMSSM the Higgs sector is more peculiar and its related collider observables are expected to give also more spectacular signatures, hence are more prone to unveil the singlet component of the Higgs potential [15]. Therefore dark matter related observables in the NMSSM are also distinct when the singlet component is at play both in the neutralino and Higgs sector. Furthermore, as far as the annihilation $\tilde{\chi}_{1}^{0} \tilde{\chi}_{1}^{0} \rightarrow \gamma \gamma\left(Z^{0}\right)$ is concerned, this observable can only be sensitive to the pseudoscalar Higgs states, since at $v=0$ scalar couplings are suppressed. In this particular case, such regions of parameter space are only reachable in the NMSSM. A noticeable example of such a mechanism is self-annihilation of dark matter particles through a s-channel resonance with a light pseudoscalar. We investigated such a mechanism with a light $\tilde{\chi}_{1}^{0}$ for the $\gamma \gamma$ final state and observed that the signal can be very bright when extremely close to the resonance. A singlino-like LSP not fulfilling this feature would on the contrary give a very low rate. These kind of highly fined-tuned scenarios would in fact be excluded taking into account the limits derived by the authors of [49]. However, if this mechanism is actually at play for the indirect detection of primaries photons, it is also the case for both the diffuse photon spectrum and relic density calculations. Indeed, if ones wants to match the CDM abundance, this fixes the pair annihilation rate to be of order the canonical value of $\langle\sigma v\rangle \sim 3 \times 10^{-27} \mathrm{~cm}^{3} / \mathrm{s}$. Interestingly, this value is in the ballpark of the FERMI sensitivity. Unfortunately, as the gamma lines is a loop suppressed process, the interesting sensitivity is one or two order of magnitude less, in the most favourable scenarios, like the pseudoscalar resonance. Outside these regions, limits on the monochromatic gamma lines are not very constraining, in particular, on the astrophysical level, with respect to the ones from dwarf spheroidal galaxies. Furthermore, even concerning the pseudoscalar resonance, the dSph put also some more stringent constraints on this mechanism, since it is also at play there. The sensitivity of experiments concerning the spectral lines still need to be improved by several orders of magnitude to be compelling. The FERMI collaboration claims that their limits on the spectral lines will be significantly improved by the time the mission is finished and we can expect a possible discovery or, in case of a null result, that more featureless regions of the NMSSM could be excluded.

\section{Acknowledgments}

The authors would like to thank G. Bélanger and F. Boudjema for bringing our attention to this topic and very instructive comments. We would also like to thank U. Nierste and P. Anghel-Vasilescu for proofreading the manuscript. GC thanks F. Domingo for helpful conversations, D. A. Vazquez for useful discussion and for providing us with points presented in [7]. The figures were created with the help of JaxoDraw [58]. This work was supported in part by the GDRI-ACPP of CNRS. The work of GC is supported by BMBF grant 05H09VKF. 


\section{A NMSSM BRST transformations for the scalar fields}

To generate the ghost Lagrangian through the BRST transformations starting from the non-linear gauge-fixing in eq. (2.1) we need, in addition to the BRST transformations for the gauge fields which can be found in [43], the BRST transformations of the scalar fields from the gauge transformation of the Higgs doublets. We can parametrise the fields of the Higgs sector in the following way

$$
H_{d}=\left(\begin{array}{c}
\left(v_{d}+\phi_{d}+i \varphi_{d}\right) / \sqrt{2} \\
-\phi_{d}^{-}
\end{array}\right), H_{u}=\left(\begin{array}{c}
\phi_{u}^{+} \\
\left(v_{u}+\phi_{u}+i \varphi_{u}\right) / \sqrt{2}
\end{array}\right), S=\left(v_{s}+\phi_{s}+i \varphi_{s}\right) / \sqrt{2}
$$

The following unitary rotations matrices turn the gauge eigenstates to the physical ones

$$
\begin{aligned}
\left(\begin{array}{l}
\phi_{1}^{ \pm} \\
\phi_{2}^{ \pm}
\end{array}\right) & =\left(\begin{array}{cc}
c_{\beta} & -s_{\beta} \\
s_{\beta} & c_{\beta}
\end{array}\right)\left(\begin{array}{c}
G^{ \pm} \\
H^{ \pm}
\end{array}\right), \\
\left(\begin{array}{c}
\phi_{1} \\
\phi_{2} \\
\phi_{s}
\end{array}\right) & =\left(\begin{array}{ccc}
Z_{11}^{h} & Z_{12}^{h} & Z_{13}^{h} \\
Z_{21}^{h} & Z_{22}^{h} & Z_{23}^{h} \\
Z_{31}^{h} & Z_{32}^{h} & Z_{33}^{h}
\end{array}\right)\left(\begin{array}{l}
H_{1} \\
H_{2} \\
H_{3}
\end{array}\right), \\
\left(\begin{array}{c}
\varphi_{1} \\
\varphi_{2} \\
\varphi_{s}
\end{array}\right) & =\left(\begin{array}{ccc}
Z_{11}^{a} s_{\beta} & Z_{21}^{a} s_{\beta} & c_{\beta} \\
Z_{11}^{a} c_{\beta} & Z_{21}^{a} c_{\beta} & -s_{\beta} \\
Z_{12}^{a} & Z_{22}^{a} & 0
\end{array}\right)\left(\begin{array}{l}
A_{1} \\
A_{2} \\
G^{0}
\end{array}\right),
\end{aligned}
$$

where we have first rotated with an angle $\beta$ in the pseudoscalar sector to single out the goldstone mode $G^{0}$ from the physical CP-odd states in eq. (A.4) [15]. As the BRST transformations are closely linked to gauge transformations we have for the singlet components,

$$
\delta_{\mathrm{BRS}} \phi_{s}=\delta_{\mathrm{BRS}} \varphi_{s}=0
$$

Provided the rotations matrices we have then a relation between the BRST transformations of the physical eigenstates once the ones for the gauge eigenstates are known,

$$
\begin{aligned}
\left(\begin{array}{c}
\delta_{\mathrm{BRS}} G^{ \pm} \\
\delta_{\mathrm{BRS}} H^{ \pm}
\end{array}\right) & =\left(\begin{array}{cc}
c_{\beta} & s_{\beta} \\
-s_{\beta} & c_{\beta}
\end{array}\right)\left(\begin{array}{c}
\delta_{\mathrm{BRS}} \phi_{1}^{ \pm} \\
\delta_{\mathrm{BRS}} \phi_{2}^{ \pm}
\end{array}\right) \\
\left(\begin{array}{c}
\delta_{\mathrm{BRS}} H_{1} \\
\delta_{\mathrm{BRS}} H_{2} \\
\delta_{\mathrm{BRS}} H_{3}
\end{array}\right) & =\left(\begin{array}{ccc}
Z_{11}^{h} & Z_{21}^{h} & Z_{31}^{h} \\
Z_{12}^{h} & Z_{22}^{h} & Z_{32}^{h} \\
Z_{13}^{h} & Z_{23}^{h} & Z_{33}^{h}
\end{array}\right)\left(\begin{array}{c}
\delta_{\mathrm{BRS}} \phi_{1} \\
\delta_{\mathrm{BRS}} \phi_{2} \\
0
\end{array}\right) \\
\left(\begin{array}{c}
\delta_{\mathrm{BRS}} A_{1} \\
\delta_{\mathrm{BRS}} A_{2} \\
\delta_{\mathrm{BRS}} G^{0}
\end{array}\right) & =\left(\begin{array}{ccc}
Z_{11}^{a} s_{\beta} & Z_{21}^{a} s_{\beta} & Z_{12}^{a} \\
Z_{21}^{a} c_{\beta} & Z_{21}^{a} c_{\beta} & Z_{22}^{a} \\
c_{\beta} & -s_{\beta} & 0
\end{array}\right)\left(\begin{array}{c}
\delta_{\mathrm{BRS}} \varphi_{1} \\
\delta_{\mathrm{BRS}} \varphi_{2} \\
0
\end{array}\right)
\end{aligned}
$$


With

$$
\begin{aligned}
\delta_{\mathrm{BRS}} \phi_{1}^{ \pm}= & \mp \frac{i g}{2} c^{ \pm}\left[v_{1}+\left(Z_{11}^{h} H_{1}+Z_{12}^{h} H_{2}+Z_{13}^{h} H_{3}\right) \mp i\left(s_{\beta}\left(Z_{11}^{a} A_{1}+Z_{21}^{a} A_{2}\right)+c_{\beta} G^{0}\right)\right] \\
& \mp i e\left(c^{A}-\frac{s_{w}^{2}-c_{w}^{2}}{2 s_{w} c_{w}}\right)\left[c_{\beta} G^{ \pm}-s_{\beta} H^{ \pm}\right] \\
\delta_{\mathrm{BRS}} \phi_{2}^{ \pm}= & \mp \frac{i g}{2} c^{ \pm}\left[v_{2}+\left(Z_{21}^{h} H_{1}+Z_{22}^{h} H_{2}+Z_{23}^{h} H_{3}\right) \pm i\left(c_{\beta}\left(Z_{11}^{a} A_{1}+Z_{21}^{a} A_{2}\right)-s_{\beta} G^{0}\right)\right] \\
& \mp i e\left(c^{A}-\frac{s_{w}^{2}-c_{w}^{2}}{2 s_{w} c_{w}}\right)\left[s_{\beta} G^{ \pm}+c_{\beta} H^{ \pm}\right] \\
\delta_{\mathrm{BRS}} \phi_{1}^{0}= & +\frac{i g}{2}\left[c_{\beta}\left(G^{-} c^{+}-G^{+} c^{-}\right)-s_{\beta}\left(H^{-} c^{+}-H^{+} c^{-}\right)\right] \\
& +\frac{e}{2 c_{w} s_{w}} c^{Z}\left[s_{\beta}\left(Z_{11}^{a} A_{1}+Z_{21}^{a} A_{2}\right)+c_{\beta} G^{0}\right] \\
\delta_{\mathrm{BRS}} \phi_{2}^{0}= & +\frac{i g}{2}\left[s_{\beta}\left(G^{-} c^{+}-G^{+} c^{-}\right)+c_{\beta}\left(H^{-} c^{+}-H^{+} c^{-}\right)\right] \\
& -\frac{e}{2 c_{w} s_{w}} c^{Z}\left[c_{\beta}\left(Z_{11}^{a} A_{1}+Z_{21}^{a} A_{2}\right)-s_{\beta} G^{0}\right] \\
\delta_{\mathrm{BRS}} \varphi_{1}^{0}= & +\frac{g}{2}\left[c_{\beta}\left(G^{-} c^{+}+G^{+} c^{-}\right)-s_{\beta}\left(H^{-} c^{+}+H^{+} c^{-}\right)\right] \\
& -\frac{e}{2 c_{w} s_{w}} c^{Z}\left[v_{1}+Z_{11}^{h} H_{1}+Z_{12}^{h} H_{2}+Z_{13}^{h} H_{3}\right] \\
\delta_{\mathrm{BRS}} \varphi_{2}^{0}= & -\frac{g}{2}\left[s_{\beta}\left(G^{-} c^{+}+G^{+} c^{-}\right)+c_{\beta}\left(H^{-} c^{+}+H^{+} c^{-}\right)\right] \\
& +\frac{e}{2 c_{w} s_{w}} c^{Z}\left[v_{2}+Z_{21}^{h} H_{1}+Z_{22}^{h} H_{2}+Z_{23}^{h} H_{3}\right]
\end{aligned}
$$

Open Access. This article is distributed under the terms of the Creative Commons Attribution Noncommercial License which permits any noncommercial use, distribution, and reproduction in any medium, provided the original author(s) and source are credited.

\section{References}

[1] The CDMS-II collaboration, Z. Ahmed et al., Dark matter search results from the CDMS II experiment, Science 327 (2010) 1619 [arXiv:0912.3592] [INSPIRE].

[2] CoGeNT collaboration, C. Aalseth et al., Results from a search for light-mass dark matter with a p-type point contact germanium detector, Phys. Rev. Lett. 106 (2011) 131301 [arXiv: 1002.4703] [INSPIRE].

[3] C. Aalseth et al., Search for an annual modulation in a p-type point contact germanium dark matter detector, Phys. Rev. Lett. 107 (2011) 141301 [arXiv:1106.0650] [INSPIRE].

[4] G. Angloher et al., Results from $730 \mathrm{~kg}$ days of the CRESST-II dark matter search, arXiv: 1109. 0702 [INSPIRE].

[5] XENON100 collaboration, E. Aprile et al., Dark matter results from 100 live days of XENON100 data, Phys. Rev. Lett. 107 (2011) 131302 [arXiv:1104.2549] [INSPIRE].

[6] R. Bernabei et al., New results from DAMA/LIBRA, Eur. Phys. J. C 67 (2010) 39 [arXiv: 1002.1028] [INSPIRE]. 
[7] D.A. Vasquez, G. Bélanger, C. Boehm, A. Pukhov and J. Silk, Can neutralinos in the MSSM and NMSSM scenarios still be light?, Phys. Rev. D 82 (2010) 115027 [arXiv:1009.4380] [INSPIRE].

[8] D.A. Vasquez, G. Bélanger and C. Boehm, Revisiting light neutralino scenarios in the MSSM, Phys. Rev. D 84 (2011) 095015 [arXiv: 1108.1338] [INSPIRE].

[9] D. Das and U. Ellwanger, Light dark matter in the NMSSM: upper bounds on direct detection cross sections, JHEP 09 (2010) 085 [arXiv: 1007.1151] [INSPIRE].

[10] P. Draper, T. Liu, C.E. Wagner, L.-T. Wang and H. Zhang, Dark light Higgs, Phys. Rev. Lett. 106 (2011) 121805 [arXiv: 1009.3963] [INSPIRE].

[11] R. Kappl, M. Ratz and M.W. Winkler, Light dark matter in the singlet-extended MSSM, Phys. Lett. B 695 (2011) 169 [arXiv:1010.0553] [InSPIRE].

[12] J.-J. Cao et al., Light dark matter in NMSSM and implication on Higgs phenomenology, Phys. Lett. B 703 (2011) 292 [arXiv:1104.1754] [InSPIRE].

[13] N. Fornengo, S. Scopel and A. Bottino, Discussing direct search of dark matter particles in the Minimal Supersymmetric extension of the Standard Model with light neutralinos, Phys. Rev. D 83 (2011) 015001 [arXiv:1011.4743] [INSPIRE].

[14] L. Calibbi, T. Ota and Y. Takanishi, Light neutralino in the MSSM: a playground for dark matter, flavor physics and collider experiments, JHEP 07 (2011) 013 [arXiv:1104.1134] [INSPIRE].

[15] U. Ellwanger, C. Hugonie and A.M. Teixeira, The next-to-minimal supersymmetric standard model, Phys. Rept. 496 (2010) 1 [arXiv:0910.1785] [inSPIRE].

[16] F. Ferrer, L.M. Krauss and S. Profumo, Indirect detection of light neutralino dark matter in the NMSSM, Phys. Rev. D 74 (2006) 115007 [hep-ph/0609257] [INSPIRE].

[17] A. Abdo et al., Fermi LAT search for photon lines from 30 to $200 \mathrm{GeV}$ and dark matter implications, Phys. Rev. Lett. 104 (2010) 091302 [arXiv:1001.4836] [INSPIRE].

[18] S. Ahlen et al., An antimatter spectrometer in space, Nucl. Instrum. Meth. A 350 (1994) 351 [INSPIRE].

[19] J. Alcaraz et.al, The Alpha Magnetic Spectrometer (AMS), Nucl. Instrum. Meth. A 478 (2002) 119.

[20] Alpha Magnetic Spectrometer, http://ams.cern.ch/.

[21] L. Bergstrom and P. Ullio, Full one loop calculation of neutralino annihilation into two photons, Nucl. Phys. B 504 (1997) 27 [hep-ph/9706232] [INSPIRE].

[22] Z. Bern, P. Gondolo and M. Perelstein, Neutralino annihilation into two photons, Phys. Lett. B 411 (1997) 86 [hep-ph/9706538] [INSPIRE].

[23] P. Ullio and L. Bergstrom, Neutralino annihilation into a photon and a $Z$ boson, Phys. Rev. D 57 (1998) 1962 [hep-ph/9707333] [INSPIRE].

[24] G. Gounaris, J. Layssac, P. Porfyriadis and F. Renard, Neutralino-neutralino annihilation to photon and gluon pairs in MSSM models, Phys. Rev. D 69 (2004) 075007 [hep-ph/0309032] [INSPIRE].

[25] F. Boudjema, A. Semenov and D. Temes, Self-annihilation of the neutralino dark matter into two photons or a $Z$ and a photon in the MSSM, Phys. Rev. D 72 (2005) 055024 [hep-ph/0507127] [INSPIRE]. 
[26] A. Djouadi et al., Benchmark scenarios for the NMSSM, JHEP 07 (2008) 002 [arXiv:0801.4321] [INSPIRE].

[27] U. Ellwanger, C.-C. Jean-Louis and A. Teixeira, Phenomenology of the general NMSSM with gauge mediated supersymmetry breaking, JHEP 05 (2008) 044 [arXiv:0803.2962] [INSPIRE].

[28] N. Baro, F. Boudjema and A. Semenov, Automatised full one-loop renormalisation of the MSSM. I. The Higgs sector, the issue of $\tan \beta$ and gauge invariance, Phys. Rev. D 78 (2008) 115003 [arXiv:0807.4668] [INSPIRE].

[29] N. Baro and F. Boudjema, Automatised full one-loop renormalisation of the MSSM II: the chargino-neutralino sector, the sfermion sector and some applications, Phys. Rev. D 80 (2009) 076010 [arXiv: 0906.1665] [inSPIRE].

[30] N. Baro, F. Boudjema and A. Semenov, Full one-loop corrections to the relic density in the MSSM: a few examples, Phys. Lett. B 660 (2008) 550 [arXiv:0710.1821] [INSPIRE].

[31] N. Baro, G. Chalons and S. Hao, Coannihilation with a chargino and gauge boson pair production at one-loop, AIP Conf. Proc. 1200 (2010) 1067 [arXiv:0909.3263] [InSPIRE].

[32] N. Baro, F. Boudjema, G. Chalons and S. Hao, Relic density at one-loop with gauge boson pair production, Phys. Rev. D 81 (2010) 015005 [arXiv:0910.3293] [INSPIRE].

[33] G. Bélanger, F. Boudjema, C. Hugonie, A. Pukhov and A. Semenov, Relic density of dark matter in the NMSSM, JCAP 09 (2005) 001 [hep-ph/0505142] [INSPIRE].

[34] F. Boudjema, L.D. Ninh, S. Hao and M.M. Weber, NLO corrections to $e^{+} e^{-} \rightarrow W W Z$ and $e^{+} e^{-} \rightarrow Z Z Z$, Phys. Rev. D 81 (2010) 073007 [arXiv:0912.4234] [INSPIRE].

[35] A. Semenov, LanHEP: a package for automatic generation of Feynman rules in gauge models, hep-ph/9608488 [INSPIRE].

[36] A. Semenov, Automatic generation of Feynman rules from the Lagrangian by means of LanHEP package, Nucl. Instrum. Meth. A 389 (1997) 293.

[37] A. Semenov, LanHEP: a package for automatic generation of Feynman rules from the Lagrangian, Comput. Phys. Commun. 115 (1998) 124 [INSPIRE].

[38] A. Semenov, LanHEP: a package for automatic generation of Feynman rules in field theory. Version 2.0, hep-ph/0208011 [INSPIRE].

[39] A. Semenov, LanHEP: a package for the automatic generation of Feynman rules in field theory. Version 3.0, Comput. Phys. Commun. 180 (2009) 431 [arXiv:0805.0555] [INSPIRE].

[40] T. Hahn and M. Pérez-Victoria, Automatized one loop calculations in four-dimensions and D-dimensions, Comput. Phys. Commun. 118 (1999) 153 [hep-ph/9807565] [InSPIRE].

[41] T. Hahn, New features in FormCalc 4, Nucl. Phys. Proc. Suppl. 135 (2004) 333 [hep-ph/0406288] [INSPIRE].

[42] F. Boudjema and E. Chopin, Double Higgs production at the linear colliders and the probing of the Higgs selfcoupling, Z. Phys. C 73 (1996) 85 [hep-ph/9507396] [INSPIRE].

[43] G. Bélanger et al., Automatic calculations in high energy physics and Grace at one-loop, Phys. Rept. 430 (2006) 117 [hep-ph/0308080] [inSPIRE].

[44] T. Hahn, LoopTools, http://www.feynarts.de/looptools/.

[45] L. Bergstrom and J. Kaplan, $\gamma$-ray lines from TeV dark matter, Astropart. Phys. 2 (1994) 261 [hep-ph/9403239] [INSPIRE]. 
[46] U. Ellwanger, J.F. Gunion and C. Hugonie, NMHDECAY: a Fortran code for the Higgs masses, couplings and decay widths in the NMSSM, JHEP 02 (2005) 066 [hep-ph/0406215] [INSPIRE].

[47] U. Ellwanger and C. Hugonie, NMHDECAY 2.0: an updated program for sparticle masses, Higgs masses, couplings and decay widths in the NMSSM, Comput. Phys. Commun. 175 (2006) 290 [hep-ph/0508022] [INSPIRE].

[48] NMSSMTools, http://www.th.u-psud.fr/NMHDECAY/nmssmtools.html.

[49] G. Vertongen and C. Weniger, Hunting dark matter $\gamma$-ray lines with the Fermi LAT, JCAP 05 (2011) 027 [arXiv: 1101.2610] [INSPIRE].

[50] J. Hisano, S. Matsumoto, M.M. Nojiri and O. Saito, Non-perturbative effect on dark matter annihilation and gamma ray signature from galactic center, Phys. Rev. D 71 (2005) 063528 [hep-ph/0412403] [INSPIRE].

[51] J.F. Gunion, D. Hooper and B. McElrath, Light neutralino dark matter in the NMSSM, Phys. Rev. D 73 (2006) 015011 [hep-ph/0509024] [InSPIRE].

[52] A.V. Belikov, J.F. Gunion, D. Hooper and T.M. Tait, CoGeNT, DAMA and light neutralino dark matter, Phys. Lett. B 705 (2011) 82 [arXiv: 1009.0549] [INSPIRE].

[53] J.F. Gunion, A.V. Belikov and D. Hooper, CoGeNT, DAMA and neutralino dark matter in the next-to-minimal supersymmetric standard model, arXiv:1009.2555 [INSPIRE].

[54] D. Albornoz Vasquez, G. Bélanger and C. Boehm, Astrophysical limits on light NMSSM neutralinos, Phys. Rev. D 84 (2011) 095008 [arXiv:1107.1614] [INSPIRE].

[55] L.E. Strigari, S.M. Koushiappas, J.S. Bullock and M. Kaplinghat, Precise constraints on the dark matter content of Milky Way dwarf galaxies for gamma-ray experiments, Phys. Rev. D 75 (2007) 083526 [astro-ph/0611925] [INSPIRE].

[56] D.T. Cumberbatch, D.E. Lopez-Fogliani, L. Roszkowski, R.R. de Austri and Y.-L.S. Tsai, Is light neutralino as dark matter still viable?, arXiv:1107.1604 [INSPIRE].

[57] A. Abdo et al., Observations of Milky Way dwarf spheroidal galaxies with the Fermi-LAT detector and constraints on Dark Matter models, Astrophys. J. 712 (2010) 147 [arXiv: 1001.4531] [INSPIRE].

[58] D. Binosi and L. Theussl, JaxoDraw: a graphical user interface for drawing Feynman diagrams, Comput. Phys. Commun. 161 (2004) 76 [hep-ph/0309015] [INSPIRE]. 